\title{
Algoritmo de Otimização do Coiote Aplicado na Identificação de um Sistema Multivariável
}

\author{
Allan Christian Krainski Ferrari*, Gideon Villar Leandro**, Leandro dos Santos Coelho***, \\ Luiz Felipe Manke****, Juliano Pierezan** \\ * Departamento de Engenharia Elétrica, UNISOCIESC, \\ Rodovia Régis Bittencourt, 18805, 81690-300 Curitiba, Paraná, Brasil \\ (e-mail: allan.ferrari@unisociesc.com.br) \\ **Departamento de Engenharia Elétrica, Universidade Federal do Paraná, \\ Caixa Postal 19011, 81531-980 Curitiba, Paraná, Brasil \\ (e-mail: gede@eletrica.ufpr.br,juliano.pierezan@ufpr.br) \\ *** Departamento de Engenharia Elétrica, Pontifícia Universidade Católica do Paraná, \\ R. Imaculada. Conceição, 1155, 80215-901, Curitiba, Paraná, Brasil \\ (e-mail: leandro.coelho@pucpr.br) \\ ****Instituição: WEG Equipamentos Elétricos S.A. - Departamento de Pesquisa e Inovação \\ Tecnológica, Av Pref. Waldemar Grubba, 3000 - Jaraguá do Sul, Santa Catarina, Brasil \\ (e-mail:luizmanke@gmail.com)
}

\begin{abstract}
The Coyote Optimization Algorithm (COA) is a recent metaheuristic that can be explored in many applications. This paper makes an application of COA in the identification process of a real multivariable system that is composed of six inputs and one output. The identification process uses a state space model in discrete time. The COA was subjected sequence of 100 runs, the quality of the estimation and validation of the models obtained by the algorithm are evaluated by the value of the multiple correction coefficient and the mean square error value. Then, the results obtained from the COA were compared with the performance of the other metaheuristics of optimization. The results show that COA can be an effective algorithm to obtain mathematical models of real multivariable systems.
\end{abstract}

\begin{abstract}
Resumo: O algoritmo de otimização do coiote (COA - Coyote Optimization Algorithm) é uma metaheurística recente que pode ser explorada em muitas aplicações. Este artigo faz uma aplicação do COA no processo de identificação de um sistema multivariável real composto por seis entradas e uma saída. Para o processo de identificação desse sistema, é utilizando um modelo de espaço de estados em tempo discreto. O COA foi submetido a uma sequência de 100 simulações, onde a qualidade da estimação e validação dos modelos obtidos pelo algoritmo são avaliados pelo valor do coeficiente de correção múltipla e o valor do erro quadrático médio. Em seguida, os resultados obtidos do COA foram comparados com o desempenho de outras metaheurísticas de otimização. Os resultados mostram que o COA pode ser um algoritmo eficaz na obtenção de modelos matemáticos de sistemas multivariáveis reais. conferência.
\end{abstract}

Keywords: coyote optimization algorithm; metaheuristics; identification; real multivariable system.

Palavras-chaves: algoritmo de otimização do coiote; metaheurísticas; identificação; sistema multivariável real.

\section{INTRODUÇÃO}

As metaheurísticas de otimização geralmente são proposstas para tentar encontrar soluções aceitáveis em problemas de otimização ou de engenharia que são considerados complexos para os métodos numéricos clássicos baseados na informação do gradiente das funções objetivo. Estes algoritmos podem ser inspirados em algum tipo de processo natural que pode ser um comportamento biológico ou um fenômeno físico (SalcedoSanz, 2016).

Os algoritmos que mimetizam um comportamento biológico podem ser classificados em dois grupos: computação evolutiva (evolutionary computing) e inteligência de enxames (swarms intelligence). As metaheurísticas pertencentes ao grupo da computação evolutiva são inspirados nas leis da evolução natural. Os algoritmos do grupo inteligência de enxames são inspirados no comportamento de certos grupos de animais que vivem de forma cooperativa (Mirjalili e Lewis, 2016).

Uma das metaheurísticas mais recentes é o algoritmo de otimização do coiote (COA - Coyote Optimization Algorithm) proposto por Pierezan e Coelho (2018). Este algoritmo é inspirado no comportamento biológico dos coiotes e o mesmo foi desenvolvido visando resolver problemas de otimização global. O COA é classificado como algoritmo de inteligência de enxames. Esta metaheurística baseia-se na estrutura social dos coiotes onde experiências são trocadas pelos mesmos. 
Este artigo tem como contribuição aplicar o algoritmo COA na identificação de um sistema multivariável real. Este tipo problema específico pode ser desafiador pois envolve a estimação e a validação de uma grande quantidade de parâmetros do sistema. A capacidade de otimização o global desta metaheurística é testada para este tipo de estudo de caso. O desempenho do COA é comparado com outras metaheurísticas bem conhecidas e aplicadas na literatura.

Este artigo está estruturado em três partes: método, resultados e conclusão. No método é realizada uma apresentação do algoritmo COA. Os resultados estão subdivididos em quatro seções. A primeira seção faz uma descrição do sistema multivariável real que é utilizado no processo de identificação. A segunda seção apresenta o modelo matemático. Na terceira seção é apresentado os critérios de análise e comparação. Na quarta seção são apresentados os resultados obtidos. Finalmente na conclusão são apresentados as restrições e os desdobramentos para trabalhos futuros.

\section{MÉTODO}

No COA, a população de coiotes é dividida em $N_{P}$ matilhas (packs), onde cada matilha contém $N_{C}$ coiotes. Neste algoritmo é assumido que a quantidade de coiotes é a mesma para todos os grupos. Logo, o tamanho da população é dado pela multiplicação de $N_{P}$ e $N_{C}$ (Pierezan e Coelho, 2018).

A condição social de cada coiote $c^{\text {th }}$ da matilha (pack) $p^{\text {th }}$ representa uma possível solução $\vec{x}$ para um problema de otimização global no instante de tempo $t^{\text {th }}$, sendo escrita por:

$$
\operatorname{soc}_{c}^{p, t}=\vec{x}=\left(x_{1}, x_{2}, \ldots, x_{D}\right)
$$

onde $D$ representa a dimensão da solução $\vec{x}$.

O COA é um algoritmo estocástico, ou seja, a população inicial de coiotes é gerada de forma aleatória com distribuição uniforme dentro de um espaço de busca definido. Logo, a condição social do coiote $c^{\text {th }}$ da matilha $p^{\text {th }}$ e da dimensão $j^{\text {th }}$ é iniciada por:

$$
\operatorname{soc}_{c, j}^{p, t}=l b_{j}+r_{j}\left(u b_{j}-l b_{j}\right)
$$

onde $l b_{j}$ e $u b_{j}$ representam respectivamente o limite inferior e superior do espaço de busca. $\mathrm{O}$ valor $r_{j}$ gera um número aleatório com distribuição uniforme dentro do intervalo $[0,1]$.

A condição social dos coiotes é avaliada pelo custo da função objetivo, sendo calculada por:

$$
f i t_{c}^{p, t}=f\left(\operatorname{soc}_{c}^{p, t}\right)
$$

Inicialmente, os coiotes são distribuídos aleatoriamente nas matilhas. Às vezes, os coiotes podem deixar suas matilhas para se tornar solitários ou podem junta-se a outras matilhas (Pitt et al., 2003). Segundo Conner et al. (2008), a evasão de um coiote de uma matilha depende do número de coiotes dentro da matilha e ocorre com probabilidade $P_{e}$, sendo determinado por:

$$
P_{e}=0,005 N_{c}^{2}
$$

A probabilidade $P_{e}$ auxilia o COA a diversificar a interação entre todos os coiotes da população, o que significa um intercâmbio cultural na população global (Pierezan e Coelho, 2018).

$\mathrm{Na}$ natureza, algumas espécies geralmente possuem dois coiotes alphas que lideram uma matilha (Conner et al., 2008). No COA, a matilha é liderada por um coiote alpha que representa a melhor solução. Para um problema de minimização, o coiote alpha da matilha $p^{\text {th }}$ no instante de tempo $t^{\text {th }}$ é definido por:

$$
\operatorname{alpha}^{p, t}=\operatorname{soc}_{c}^{p, t} \mid \arg _{C=1,2, \ldots N_{C}} \min \left(f\left(\operatorname{soc}_{c}^{p, t}\right)\right)
$$

O COA assume que os coiotes são suficientemente organizados para compartilhar as condições sociais e contribuir para a manutenção da matilha. Assim, o COA vincula todas as informações dos coiotes e determina a tendência cultural da matilha, sendo calculado por:

$$
\text { cult }_{j}^{p, t}=\left\{\begin{array}{lc}
O_{\frac{\left(N_{C}+1\right)}{2}, j}^{p, t}, & N_{C} \\
O_{\frac{\left(N_{C}\right)}{2}, j}^{p, t}+O_{\frac{\left(N_{C}+1\right)}{2}, j}^{p, t}, & \text { émpar } \\
2 & \text { Contrório }
\end{array}\right.
$$

onde $O^{p, t}$ representa as condições sociais classificadas de todos os coiotes da matilha $p^{\text {th }}$ no instante de tempo $t^{\text {th }}$ para todo $j$ no intervalo $[1, D]$. Em outras palavras, a tendência cultural da matilha é calculada como a mediana das condições sociais de todos os coiotes de uma específica matilha (Pierezan e Coelho, 2018).

Os dois principais eventos biológicos na vida de um animal são o nascimento e a morte, o COA calcula a idade dos coiotes em anos, sendo denotado como $a g e_{c}^{p, t}$. O nascimento de novos coiotes é escrito como a combinação das condições sociais de dois pais (escolhidos aleatoriamente) e mais a influência do meio ambiente, sendo representado por:

$$
\operatorname{pup}_{j}^{p, t}=\left\{\begin{array}{lr}
\operatorname{soc}_{r_{1}, j}^{p, t}, & \operatorname{rnd}_{j}<P_{S} \text { ou } j=j_{1} \\
\operatorname{soc}_{r_{2}, j}^{p, t}, & r n d_{j} \geq P_{S}+P_{a} \text { ou } j=j_{2} \\
R_{j}, & \text { Caso Contrário }
\end{array}\right.
$$

onde $r_{1}$ e $r_{2}$ são coiotes escolhidos de forma aleatória na matilha $p^{\text {th }}, j_{1}$ e $j_{2}$ são duas dimensões aleatórias do problema, $P_{S}$ é a probabilidade de dispersão, $P_{a}$ é a probabilidade de associação, $R_{j}$ é um número aleatório dentro da variável de decisão ligada a dimensão $j$ e $r n d_{j}$ é um número gerado de forma aleatória com distribuição uniforme dentro do intervalo [0, 1] (Pierezan e Coelho, 2018).

A probabilidade de dispersão e de associação norteiam a diversidade cultural dos coiotes na matilha. No COA, o $P_{S}$ e o $P_{a}$ foram definidos como:

$$
\begin{gathered}
P_{S}=\frac{1}{D} \\
P_{a}=\frac{1-P_{S}}{2}
\end{gathered}
$$

onde $P_{a}$ estabelece o mesmo impacto de influência para ambos os pais (Pierezan e Coelho, 2018). 
Para manter o tamanho da população estática, o COA sincroniza o nascimento e a morte dos coiotes. Este mecanismo está descrito no Algoritmo 1, onde $\omega$ representa o grupo de coiotes que é pior adaptado ao meio ambiente do que o filhote e $\varphi$ representa o número de coiotes neste grupo. Se dois ou mais coiotes têm idade semelhante (na linha 4), então o coiote menos adaptado é aquele que morre.

\begin{tabular}{ll}
\hline Algoritmo 1: Nascimentos e morte dentro da matilha \\
\hline 1: & Calcula $\omega$ e $\varphi$ \\
2: & Se $\varphi=1$, Então \\
3: & O filhote sobrevive e um único coiote em $\omega$ morre. \\
4: & Senão Se $\varphi>1$, Então \\
5: & O filhote sobrevive e o coiote mais velho em $\omega$ morre. \\
6: & Senão \\
7: & O filhote morre. \\
8: & Fim Se \\
\hline
\end{tabular}

Para representar a interação cultural dentro das matilhas no COA, é assumido que os coiotes estão sobre a influência de um coiote alpha e sobre influência da matilha. $\mathrm{O}$ valor de $\delta_{1}$ corresponde à diferença entre o coiote alpha e um coiote $\left(c r_{1}\right)$. $\mathrm{O}$ valor de $\delta_{2}$ corresponde à diferença entre a tendência cultural da matilha e um coiote $\left(c r_{2}\right)$. Os coiotes $c r_{1}$ e $c r_{2}$ são escolhidos dentro da matinha de forma aleatória com distribuição uniforme, os valores $\delta_{1}$ e $\delta_{2}$ são escritos respectivamente por:

$$
\begin{array}{r}
\delta_{1}=\operatorname{alpha}^{p, t}-\operatorname{soc}_{c r_{1}}^{p, t} \\
\delta_{2}=\operatorname{cult}^{p, t}-\operatorname{soc}_{c r_{2}}^{p, t}
\end{array}
$$

O novo valor da condição social dos coiotes é atualizado em função do coiote alpha e da influência da matilha, como segue a equação:

$$
n e w_{-} \operatorname{soc}_{c}^{p, t}=\operatorname{soc}_{c}^{p, t}+r_{1} \delta_{1}+r_{2} \delta_{2}
$$

onde $r_{1}$ e $r_{2}$ são respectivamente os pesos do coiote alpha e da influência da matilha, estes pesos são valores gerados de forma aleatória com distribuição uniforme dentro intervalo [0, 1]. A nova condição social é avaliada por:

$$
n e w_{-} f i t_{c}^{p, t}=f\left(n e w_{-} s o c_{c}^{p, t}\right)
$$

A capacidade cognitiva do coiote de decidir se a nova social condição é melhor ou não do que a anterior, sendo calculado por:

$$
\operatorname{soc}_{c}^{p, t+1}=\left\{\begin{array}{l}
\operatorname{new}_{-} \operatorname{soc}_{c}^{p, t}, \quad \text { new_fit } t_{c}^{p, t}<f i t_{c}^{p, t} \\
\operatorname{soc}_{c}^{p, t}, \quad \text { Caso contrário }
\end{array}\right.
$$

Finalmente, a condição social do coiote melhor adaptado no meio ambiente é selecionado como solução global do problema. O pseudocódigo do COA está descrito no Algoritmo 2.

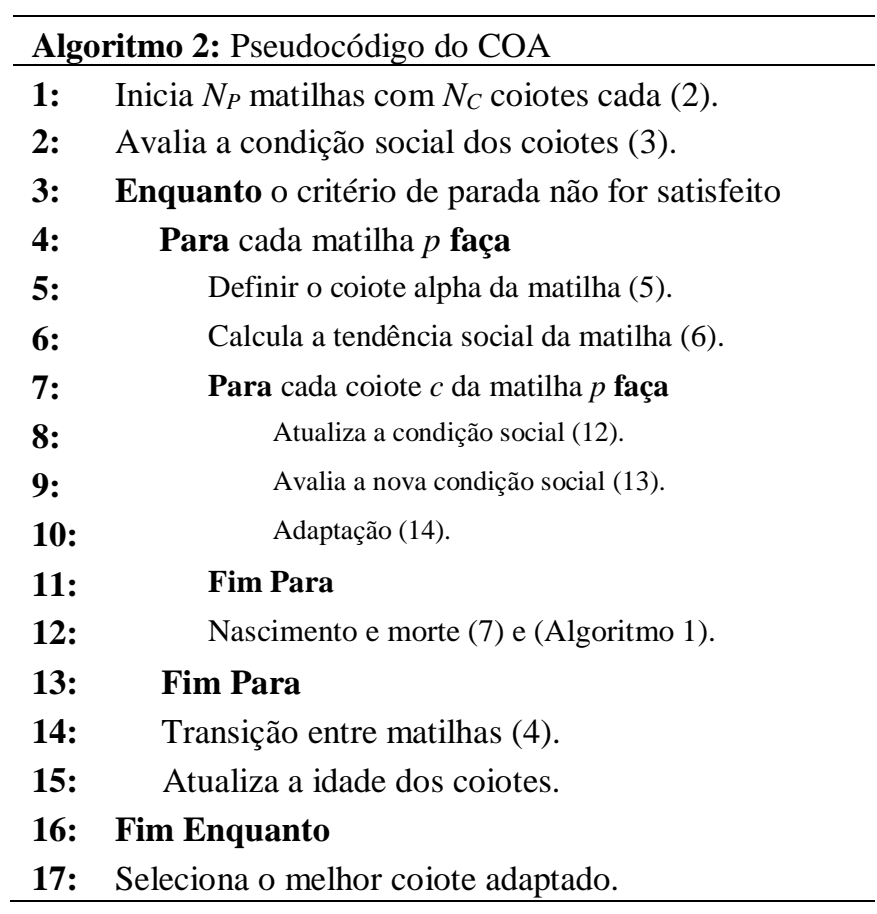

\section{ANÁLISE DE RESULTADOS}

Para avaliar a capacidade de otimização global do algoritmo COA, o mesmo foi utilizado no processo de identificação de um sistema multivariável real. O algoritmo COA foi comparado com outros três algoritmos que são: algoritmo de pastoreio do elefante (EHO - Elephant Herding Optimization); otimização por enxame de partículas (PSO Particle Swarm Optimization) e evolução diferencial (DE Differential Evolution). As metaheurísticas PSO e DE são bem conhecidas na literatura, já o algoritmo EHO é recente e foi proposto por Wang et al. (2016). O algoritmo do elefante possui uma estrutura social semelhante ao COA, onde a população também é dividida em grupos (clãs).

\subsection{Descrição do Sistema Multivariável}

Este trabalho utiliza um sistema multivariável real constituído de seis entradas e uma saída, o mesmo é utilizado para analisar as metaheurísticas. As variáveis de entrada são valores de corrente elétrica controladas por meio de modulação PWM (Pulse Width Modulation) que controlam as bombas que alimentam um sistema de distribuição de água. O valor da variável de saída é obtido através do sensor de vazão que mede o volume de água em relação ao tempo sendo fornecida por seis bombas.

Na Fig. 1 estão representados os dados utilizados na estimação e validação dos parâmetros do modelo matemático de (15). A estimação utiliza uma amostra de 115 elementos, enquanto que a validação utiliza uma amostra de 80 elementos. Cada elemento da amostra contém os valores das seis entradas e da única saída para um determinado tempo. Estes dados possuem tempo de amostragem igual a uma hora e foram fornecidos pela Companhia Paranaense de Saneamento (SANEPAR, 2014). Estes dados foram utilizados em Ferrari et al. (2018). 

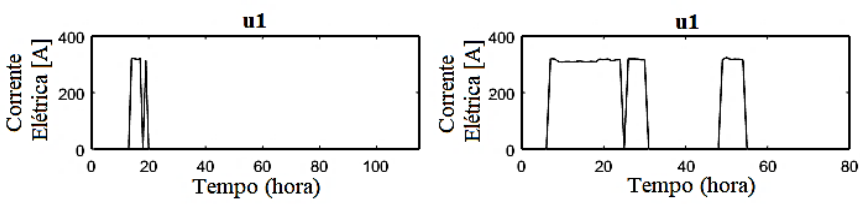

$\mathbf{u} 2$

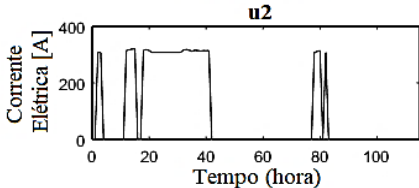

u3

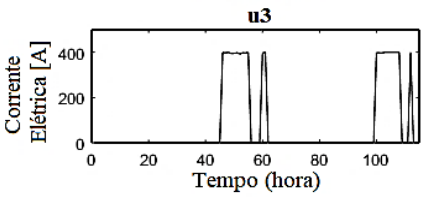

14

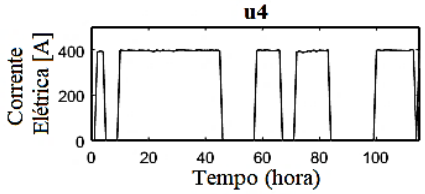

u5

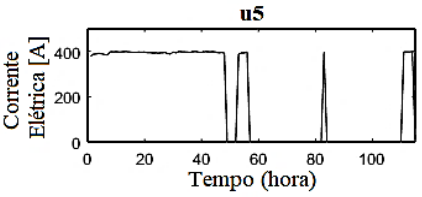

u6

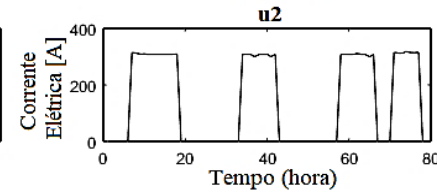

u3

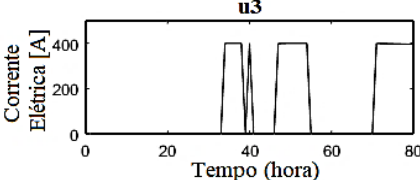

u4

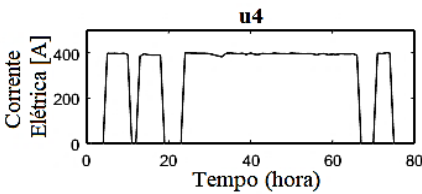

u5

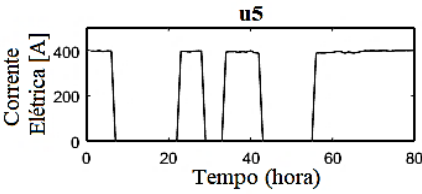

u6

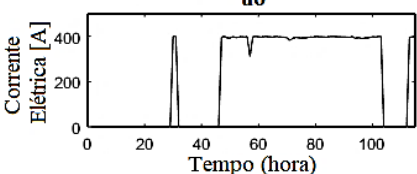

$\mathbf{y}$

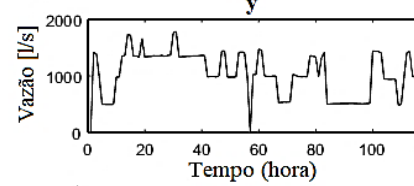

(a)
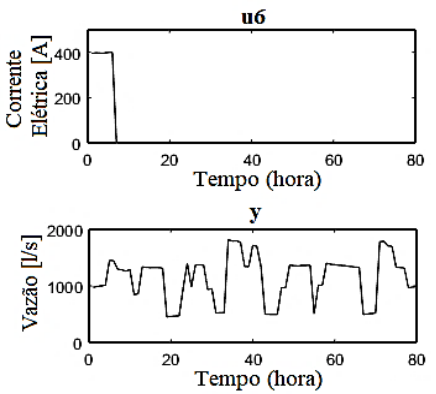

(b)

Fig. 1 Dados do sistema multivariável: (a) estimação e (b) validação.

O sistema multivariável utilizado no processo de identificação é denominado de sistema MISO (Multiple Inputs and Single Output). Para o processo de identificação, os sistemas multivariáveis podem ser considerados desafiadores para a obtenção de um modelo matemático adequado. Isto se deve à variação no comportamento desses sistemas que podem depender da interação simultânea das suas variáveis (Goodwin et al., 2000).

\subsection{Modelo Matemático}

O processo de identificação deste trabalho utiliza um modelo do tipo caixa preta. O modelo do tipo caixa preta é empregado quando conhece somente os dados de entrada e de saída do sistema a ser modelado matematicamente (Aguirre, 2004). O modelo matemático adotado está representado em espaço de estados em tempo discreto. Este modelo foi o mesmo utilizado em Ferrari et al. (2018).

$$
\begin{aligned}
x[k+1] & =G x[k]+H u[k] \\
y[k] & =C x[k]+D u[k]
\end{aligned}
$$

onde:

$$
\begin{aligned}
& x[k+1]=\left[\begin{array}{l}
x_{1}[k+1] \\
x_{2}[k+1] \\
x_{3}[k+1]
\end{array}\right]_{3 \times 1}, x[k]=\left[\begin{array}{l}
x_{1}[k] \\
x_{2}[k] \\
x_{3}[k]
\end{array}\right]_{3 \times 1}, \\
& G=\left[\begin{array}{lll}
0 & 0 & -a_{3} \\
1 & 0 & -a_{2} \\
0 & 1 & -a_{1}
\end{array}\right]_{3 \times 3}, C=\left[\begin{array}{lll}
0 & 0 & 1
\end{array}\right]_{1 \times 3}, \\
& H=\left[\begin{array}{lll}
\left(b_{31}-a_{3} b_{01}\right) & \left(b_{32}-a_{3} b_{02}\right) & \left(b_{33}-a_{3} b_{03}\right) \\
\left(b_{21}-a_{2} b_{01}\right) & \left(b_{22}-a_{2} b_{02}\right) & \left(b_{23}-a_{2} b_{03}\right) \\
\left(b_{11}-a_{1} b_{01}\right) & \left(b_{12}-a_{1} b_{02}\right) & \left(b_{13}-a_{1} b_{03}\right)
\end{array}\right. \\
& \begin{array}{lll}
\left(b_{34}-a_{3} b_{04}\right) & \left(b_{35}-a_{3} b_{05}\right)\left(b_{36}-a_{3} b_{06}\right)
\end{array} \\
& \text { … } \quad \begin{array}{llll}
\left.b_{24}-a_{2} b_{04}\right) & \left(b_{25}-a_{2} b_{05}\right) & \left(b_{26}-a_{2} b_{06}\right)
\end{array} \quad e \\
& \left.\begin{array}{llll}
\left(b_{14}-a_{1} b_{04}\right) & \left(b_{15}-a_{1} b_{05}\right) & \left(b_{16}-a_{1} b_{06}\right)
\end{array}\right]_{3 \times 6} \\
& D=\left[\begin{array}{llllll}
b_{01} & b_{02} & b_{03} & b_{04} & b_{05} & b_{06}
\end{array}\right]_{1 \times 6}
\end{aligned}
$$

A equação (15) é a função objetivo dos algoritmos, onde os coeficientes $a_{i}$ e $b_{i j}$ das matrizes $G, H, C$ e $D$ serão os parâmetros a serem estimados. As seis entradas são representadas por $\mathrm{u}[\mathrm{k}]$ e sua respectiva resposta por $\mathrm{y}[\mathrm{k}]$.

\subsection{Critérios de Análise e Comparação}

As metaheurísticas utilizadas neste trabalho foram submetidas a uma série de 100 simulações visando avaliar a qualidade da estimação e validação dos parâmetros de (15). Nas etapas de estimação e validação, foi adotado o valor MSE (Mean of Squared Error) e o coeficiente de correlação múltipla $R^{2}$ como critérios para avaliar a qualidade dos modelos matemáticos obtidos. Para critério de parada dos algoritmos, foi estabelecido um número máximo de 500 iterações. Os valores obtidos do processo de identificação $\left(R^{2}\right.$ e MSE) estão representados por meio de média $\vec{\mu}$ e desvio-padrão $\sigma$.

Para que a comparação e análise dos algoritmos seja a mais justa possível, foi estabelecido para cada algoritmo um tamanho de população $N_{P}$ igual a 100 indivíduos. Também foi definida a região de busca que compreende o intervalo $[-1,1]$. Neste trabalho, a população do algoritmo COA foi dividida em

\begin{tabular}{|c|c|c|}
\hline \multirow{2}{*}{ Algoritmos } & \multicolumn{2}{|c|}{ Parâmetros de controle } \\
\hline & Fixo & Variável \\
\hline $\mathrm{EHO}$ & $\begin{array}{c}\text { Número de clãs }(\boldsymbol{n C l a n}): \\
n \text { Clan }=5 . \\
\text { Número de elefantes por clã }(\text { nci }): \\
n c i=20 . \\
\text { Fator de influência da } \\
\text { matriarca }(\boldsymbol{\alpha}): \alpha=0,7 . \\
\text { Fator de influência da posição } \\
\text { do centro do clã }(\boldsymbol{\beta}): \beta=0,6 .\end{array}$ & Não tem. \\
\hline
\end{tabular}
5 matilhas, ou seja, cada matilha ficou composta por 20 coiotes, o que resulta em uma população total de 100 indivíduos. Na Tabela 1 está representado os parâmetros de controle dos algoritmos utilizados na comparação com o COA no processo de identificação do sistema multivariável. Neste trabalho, os parâmetros de controle fixo da Tabela 1 foram ajustados de maneira empírica.

Tabela 1: Parâmetros das metaheurísticas EHO, PSO e DE. 


\begin{tabular}{|c|c|c|}
\hline \multirow{2}{*}{ Algoritmos } & \multicolumn{2}{|c|}{ Parâmetros de controle } \\
\hline & Fixo & Variável \\
\hline PSO & $\begin{array}{c}\text { Constante de aceleração }(\boldsymbol{\beta}) \text { : } \\
\qquad \beta=0,3\end{array}$ & $\begin{array}{c}\text { Constante de } \\
\text { aceleração }(\alpha): \\
\alpha=\alpha \cdot 0,99999999^{t} \text {. } \\
\text { Valor inicial: } \alpha=0,1 . \\
\alpha-\text { decresce de forma } \\
\text { exponencial no } \\
\text { decorrer da iteração } t\end{array}$ \\
\hline $\mathrm{DE}$ & $\begin{array}{c}\text { Versão: DE/Best/1/Bin } \\
\text { Taxa de mutação }(\boldsymbol{F}): F=0,75 \text {. } \\
\text { Taxa de cruzamento }\left(\boldsymbol{C}_{\boldsymbol{R}}\right): C_{R}=0,25\end{array}$ & Não tem. \\
\hline
\end{tabular}

O teste de hipóteses foi adotado como método estatístico para comparar o desempenho do algoritmo COA em relação as outras metaheurísticas. $\mathrm{O}$ teste de hipóteses consiste em fazer uma comparação do valor de $z_{t}$ que está associado à hipótese alternativa $\left(\mathrm{H}_{1}\right)$ e o valor da estatística do erro de confiança $z_{\alpha}$ está associado à hipótese nula $\left(\mathrm{H}_{0}\right)$. Esta comparação é realizada através do cálculo da probabilidade que é obtida pela curva de distribuição normal (Montgomery e Runger, 2012).

O teste de hipóteses foi aplicado para duas amostras populacionais diferentes com valores de média e desviopadrão conhecidos, com um nível de confiança igual a $99 \%$. $\mathrm{O}$ valor estatístico teste $z_{t}$ é obtido por:

$$
z_{t}=\frac{\left(\vec{\mu}_{1}-\vec{\mu}_{2}\right)}{\sqrt{\frac{\sigma_{1}^{2}}{n_{1}}-\frac{\sigma_{2}^{2}}{n_{2}}}}
$$

onde $\vec{\mu}_{1}, \sigma_{1}$ e $n_{1}$ são respectivamente a média, o desvio-padrão e o número de elementos da $1^{a}$ amostra. Enquanto $\vec{\mu}_{2}, \sigma_{2}$ e $n_{2}$ são respectivamente a média, o desvio-padrão e o número de elementos da $2^{\mathrm{a}}$ amostra (Montgomery e Runger, 2012).

O teste de hipóteses foi realizado com uma confiabilidade de $99 \%$, ou seja, $z_{t}$ deve ser igual a 2,37. Para esta condição o teste de hipóteses terá direção unidirecional à direita $\left(z_{t}>\right.$ $z_{\alpha}$ ). Se o valor de $z_{t}$ for maior que $z_{\alpha}$, então $\mathrm{H}_{1}$ é aceita. Se $z_{t}$ for menor que $z_{\alpha}$, então $\mathrm{H}_{0}$ é aceita. Neste trabalho, o teste de hipóteses fez a comparação de dois algoritmos por vez, para $\mathrm{H}_{0}$ foi considerado o algoritmo com menor valor médio de $R^{2}$ obtido na validação (a pior solução) e para $\mathrm{H}_{1}$ o algoritmo que supostamente converge melhor para uma solução com maior valor médio de $R^{2}$ obtido na validação (a melhor solução).

Para uma confiabilidade de $99 \%$ : se $\mathrm{H}_{1}$ for aceito, significa que o algoritmo com maior valor médio de $R^{2}$ converge para uma solução melhor em relação ao algoritmo com o menor valor médio, sendo este representado por $\mathrm{H}_{0}$; caso contrário, estatisticamente o algoritmo representado por $\mathrm{H}_{1}$ não pode ser considerado melhor que $\mathrm{H}_{0}$. Para esta pesquisa foi utilizado o software Matlab® na programação das metaheurísticas.

\subsection{Resultados do Algoritmo COA}

Os resultados do COA e das outras metaheurísticas obtidos no processo de identificação do sistema multivariável estão representados a seguir.

Nas Figs. 2 e 3 aparecem respectivamente os resultados da estimação e validação dos algoritmos COA, EHO, PSO e DE. Esses algoritmos conseguiram representar de forma adequada o modelo multivariável real. Para práticas de controle, um modelo matemático que represente um processo real de forma adequada deve apresentar um valor de $R^{2}$ entre 0,9 a 1 (Coelho e Coelho, 2000).

Pode-se observar nos resultados obtidos para 100 simulações (Tabela 2, Tabela 3, Tabela 4 e Fig. 3) que o COA se mostrou eficiente na modelagem do sistema multivariável do tipo MISO.

Tabela 2: Desempenho dos algoritmos para 100 simulações.

\begin{tabular}{|c|c|c|c|c|c|}
\hline \multirow{2}{*}{\multicolumn{2}{|c|}{ Dados obtidos }} & \multicolumn{4}{|c|}{ Algoritmos } \\
\hline & & \multirow{2}{*}{$\begin{array}{r}\mathrm{COA} \\
6,42 \mathrm{e} 3\end{array}$} & \multirow{2}{*}{$\begin{array}{c}\text { EHO } \\
9,81 \mathrm{e} 3\end{array}$} & \multirow{2}{*}{$\begin{array}{c}\text { PSO } \\
9,13 \mathrm{e} 3\end{array}$} & \multirow{2}{*}{$\begin{array}{c}\mathrm{DE} \\
5,04 \mathrm{e} 3 \\
\end{array}$} \\
\hline & $\vec{\mu}$ & & & & \\
\hline (Estimação) & $\sigma$ & 727,60 & 572,96 & $1,36 \mathrm{e} 3$ & 594,43 \\
\hline \multirow{2}{*}{$\begin{array}{c}M S E \\
\text { (Validação) } \\
\end{array}$} & $\vec{\mu}$ & $1,57 \mathrm{e} 4$ & $1,28 \mathrm{e} 4$ & $1,88 \mathrm{e} 4$ & $1,13 \mathrm{e} 4$ \\
\hline & $\sigma$ & $2,64 \mathrm{e} 3$ & $1,21 \mathrm{e} 3$ & $5,76 \mathrm{e} 3$ & $2,36 \mathrm{e} 3$ \\
\hline \multirow{2}{*}{$\begin{array}{c}R^{2} \\
\text { (Estimação) }\end{array}$} & $\vec{\mu}$ & 0,9584 & 0,9364 & 0,9408 & 0,9673 \\
\hline & $\sigma$ & 0,0047 & 0,0037 & 0,0089 & 0,0039 \\
\hline \multirow{2}{*}{$\begin{array}{c}R^{2} \\
\text { (Validação) }\end{array}$} & $\vec{\mu}$ & 0,9011 & 0,9190 & 0,8810 & 0,9291 \\
\hline & $\sigma$ & 0,0167 & 0,0076 & 0,0363 & 0,0148 \\
\hline \multicolumn{2}{|c|}{$\begin{array}{c}M S E \\
\text { (Melhor modelo da } \\
\text { validação) }\end{array}$} & $1,08 \mathrm{e} 4$ & $1,03 \mathrm{e} 4$ & $7,78 \mathrm{e} 3$ & $7,87 \mathrm{e} 3$ \\
\hline \multicolumn{2}{|c|}{$\begin{array}{c}R^{2} \\
\text { (Melhor modelo da } \\
\text { validação) }\end{array}$} & 0,9316 & 0,9349 & 0,9510 & 0,9504 \\
\hline
\end{tabular}

Observa-se na Tabela 2 que o algoritmo COA se mostrou competitivo em relação aos demais na estimação e validação dos parâmetros obtidos através de (15). No processo de estimação, o COA foi inferior ao $\mathrm{DE}$ em função do valor médio obtido do MSE e $R^{2}$, enquanto que no processo de validação, o COA foi superior apenas ao PSO.

Na Tabela 3 estão representados os melhores parâmetros obtidos pelos algoritmos no processo de validação, esses parâmetros foram utilizados para gerar os gráficos das Figs. 2 e 3. No teste de hipóteses (Tabela 4), o algoritmo COA foi o terceiro melhor, sendo superior apenas ao PSO.

Tabela 3: Os parâmetros estimados do melhor modelo obtido por cada algoritmo para 100 simulações.

\begin{tabular}{|c|c|c|c|c|}
\hline \multirow{2}{*}{$\begin{array}{c}\text { Parâmetros } \\
\text { Estimados }\end{array}$} & \multicolumn{4}{|c|}{ Algoritmos } \\
\cline { 2 - 5 } & COA & EHO & PSO & ED \\
\hline$a_{1}$ & $-0,3120$ & 0,6981 & 0,0282 & 0,1982 \\
\hline$a_{2}$ & 0,7473 & 0,7607 & 0,1117 & 0,4754 \\
\hline$a_{3}$ & $-0,1202$ & 0,6557 & 0,1807 & 0,2029 \\
\hline$b_{01}$ & 1,0000 & 1,0267 & 1,1037 & 1,2728 \\
\hline$b_{11}$ & $-0,2816$ & 0,8753 & 0,1116 & 0,1354 \\
\hline$b_{21}$ & 0,8498 & 1,0182 & 0,4236 & 0,7065 \\
\hline$b_{31}$ & 0,0181 & 0,9137 & 0,2100 & 0,2191 \\
\hline$b_{02}$ & 0,9858 & 1,0298 & 0,9237 & 1,1490 \\
\hline$b_{12}$ & $-0,1856$ & 0,9439 & $-0,3752$ & 0,3101 \\
\hline$b_{22}$ & 0,7567 & 0,9415 & 0,6524 & 0,6183 \\
\hline$b_{32}$ & $-0,0368$ & 0,8815 & 0,1149 & 0,1853 \\
\hline$b_{03}$ & 0,9866 & 1,0219 & 1,1057 & 1,1652 \\
\hline$b_{13}$ & $-0,0697$ & 0,9581 & $-0,1896$ & 0,3063 \\
\hline$b_{23}$ & 0,4444 & 0,8062 & 0,3622 & 0,4947 \\
\hline$b_{33}$ & $5,35 \mathrm{e}-4$ & 0,8343 & 0,2870 & 0,2211 \\
\hline$b_{04}$ & 1,0000 & 1,0491 & 1,4046 & 1,2178 \\
\hline$b_{14}$ & $-0,0865$ & 0,9656 & 0,1576 & 0,2964 \\
\hline$b_{24}$ & 0,9028 & 0,9332 & $-0,0117$ & 0,5515 \\
\hline & & & & \\
\hline
\end{tabular}




\begin{tabular}{|c|c|c|c|c|}
\hline$b_{34}$ & $-0,0539$ & 0,8239 & 0,3177 & 0,2896 \\
\hline$b_{05}$ & 1,0000 & 1,0535 & 1,2640 & 1,2988 \\
\hline$b_{15}$ & $-0,3218$ & 0,8436 & $-0,2316$ & $-0,0936$ \\
\hline$b_{25}$ & 1,0000 & 0,8684 & 0,1825 & 0,6966 \\
\hline$b_{35}$ & $-0,1337$ & 0,9635 & 0,2106 & 0,2971 \\
\hline$b_{06}$ & 1,0000 & 1,0271 & 1,4692 & 1,1938 \\
\hline$b_{16}$ & $-0,0609$ & 0,9995 & $-0,6464$ & 0,2669 \\
\hline$b_{26}$ & 0,7296 & 0,9775 & 0,7262 & 0,5842 \\
\hline$b_{36}$ & 0,0140 & 0,8142 & 0,1416 & 0,2172 \\
\hline
\end{tabular}

Tabela 4: Teste de hipóteses para o valor de $\boldsymbol{R}^{2}$ obtido na validação para 100 simulações.

\begin{tabular}{|c|c|c|c|}
\hline \multicolumn{5}{|c|}{ Teste de Hipóteses } \\
Se: $z_{t} \geq z_{\alpha}$, logo: aceita (A) $\mathrm{H}_{1}$ e rejeita (R) $\mathrm{H}_{0}$. \\
Se: $z_{t}<z_{\alpha}$, logo: aceita (A) $\mathrm{H}_{0}$ e rejeita (R) $\mathrm{H}_{1}$. \\
\hline Comparação entre algoritmos & $z_{t}$ & $\mathrm{H}_{0}:$ & $\mathrm{H}_{1}:$ \\
\hline $\mathrm{H}_{0}$ : COA e $\mathrm{H}_{1}:$ EHO. & 9,7558 & $\mathrm{R}$ & $\mathrm{A}$ \\
\hline $\mathrm{H}_{0}:$ PSO e $\mathrm{H}_{1}$ : COA. & 5,0304 & $\mathrm{R}$ & $\mathrm{A}$ \\
\hline $\mathrm{H}_{0}$ : COA e $\mathrm{H}_{1}$ : DE. & 12,5480 & $\mathrm{R}$ & $\mathrm{A}$ \\
\hline
\end{tabular}

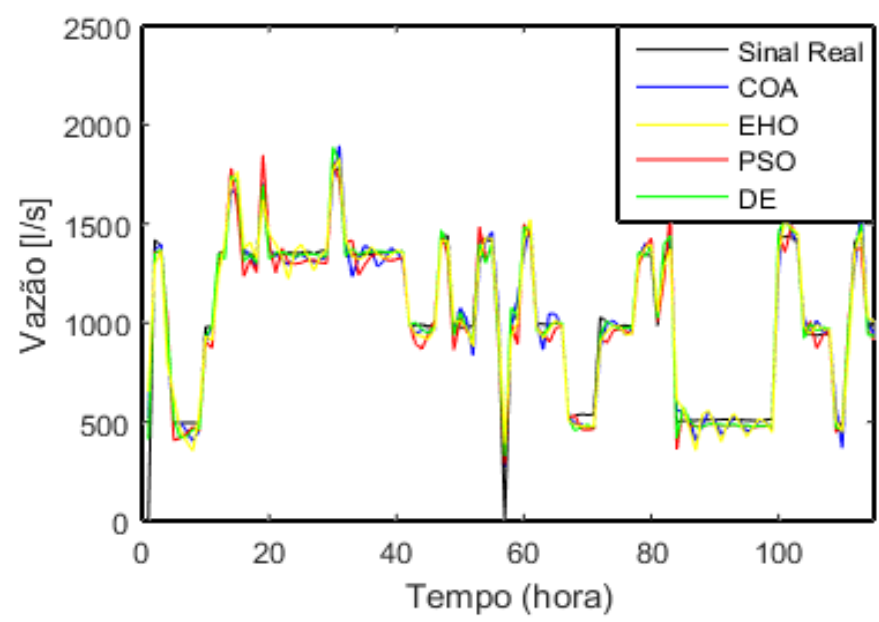

Fig. 2 Resultado da estimação do sistema multivariável a partir do melhor $R^{2}$ obtido na validação.

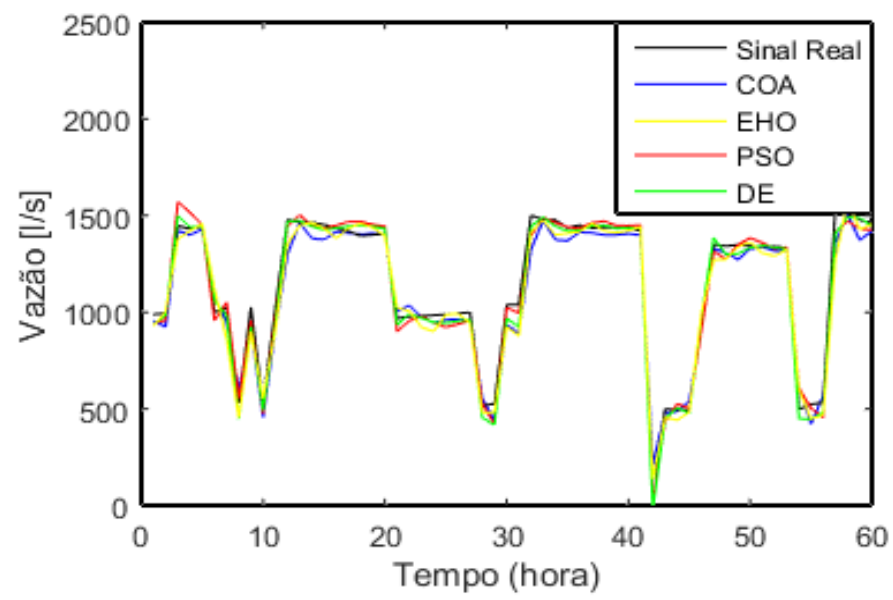

Fig. 3 Resultado da validação do sistema multivariável a partir do melhor $R^{2}$ obtido na validação.

\section{CONCLUSÕES}

O algoritmo COA é uma das metaheurísticas mais recentes na literatura que foi desenvolvida visando resolver problemas de otimização global. Para o processo de identificação de um sistema multivariável que envolve a estimação de muitas variáveis, o algoritmo COA mostrou-se eficiente o suficiente para obtenção de um modelo matemático que seja usado para práticas de controle. Para este tipo de aplicação, a capacidade de otimização global do algoritmo COA pode ser melhorada aumentando o número de coiotes da população ou o número de matilhas. Logo, pode-se obter modelos matemáticos mais precisos.

Neste trabalho, foi mostrado que o desempenho do COA pode ser competitivo quando comparado com outros algoritmos. O COA por ser uma metaheurística recente, certamente outros autores poderão propor novas variantes do mesmo com a finalidade de melhorar as suas propriedades ou explorar outras aplicações que poderão proporcionar resultados melhores em relação as outras metaheurísticas.

\section{REFERÊNCIAS}

Aguirre, L. A. (2004). Introdução à Identificação de Sistemas: Técnicas Lineares e Não Lineares Aplicadas a Sistemas Reais. Belo Horizonte, MG, Editora UFMG.

Coelho, L. S. e Coelho, A. R. (2000). Estudo comparativo de configurações de algoritmos genéticos aplicados à identificação de processo multivariável. Anais do XIII Congresso Brasileiro de Automática - CBA 2000, Florianópolis, Brasil, pp. 1-6.

Conner, M. M., Ebinger, M. R. and Knowlton, F. F. (2008). Evaluating coyote management strategies using a spatially explicit, individual-based, socially structured population model, Ecological Modelling, 219(1), pp. 234-247.

Ferrari, A. C. K., Leandro, G. V., Coelho, L. S., Silva, C. A. G., Osinski, C. and Pelacini, D. A. F. (2018). Algoritmo de pastoreio do elefante aplicado na identificação de um sistema multivariável, INDUSCON 2018 13th IEEE/IAS International Conference, São Paulo, Brasil, pp. 1-6.

Goodwin, G. C., Graebe, S. F. and Salgado, M. E. (2000). Control System Design, Pearson, Valparaiso, Chile.

Mirjalili, S. and Lewis, A. (2016). The whale optimization algorithm, Advances in Engineering Software, 95, pp. 51-67.

Montgomery, D. C. and Runger, G. C. (2012). Estatística Aplicada e Probabilidade para Engenheiros, LTC, $5^{\mathrm{a}}$ ed.

Pierezan, J. and Coelho, L. D. S. (2018). Coyote optimization algorithm: A new metaheuristic for global optimization problems, 2018 IEEE Congress on Evolutionary Computation (CEC), Rio de Janeiro, Brazil, pp. 26332640.

Pitt, W. C., Box, P. W. and Knowlton, F. (2003). An individual-based model of canid populations: modelling territoriality and social structure, Ecological Modelling, 166(1), pp. 109-121.

Salcedo-Sanz, S. (2016). Modern meta-heuristics based on nonlinear physics processes: A review of models and design procedures, Physics Reports, 655, pp. 1-70.

SANEPAR (2014). Dados do sistema de reservatório Tarumã. Curitiba, PR, Brasil.

Wang, G.-G., Deb, S., Gao, X.-Z. and Coelho, L. S. (2016). A new metaheuristic optimization algorithm motivated by elephant herding behaviour, International Journal of BioInspired Computation (IJBIC), 8(6), pp. 394-409. 